\title{
SALT spectroscopic observations of galaxy clusters detected by ACT and a Type II quasar hosted by a brightest cluster galaxy
}

\author{
Matt Hilton* \\ Astrophysics \& Cosmology Research Unit, School of Mathematics, Statistics \& Computer \\ Science, University of KwaZulu-Natal, Westville Campus, Durban 4041, ZA \\ E-mail: hiltonmeukzn.ac.za
}

\begin{abstract}
We present SALT follow-up observations of seven massive clusters detected by the Atacama Cosmology Telescope (ACT) on the celestial equator using the Sunyaev-Zel'dovich (SZ) effect. We conducted multi-object spectroscopic observations with the Robert Stobie Spectrograph in order to measure galaxy redshifts in each cluster field, determine the cluster line-of sight velocity dispersions, and infer the cluster dynamical masses. We find that the clusters, which span the redshift range $0.3<z<0.55$, range in mass from $(5-20) \times 10^{14} \mathrm{M}_{\odot}\left(M_{200 c}\right)$. Their masses, given their SZ signals, are similar to those of southern hemisphere ACT clusters previously observed using Gemini and the VLT. We note that the brightest cluster galaxy in one of the systems studied, ACT-CL J0320.4+0032 at $z=0.38$, hosts a Type II quasar. Only a handful of such systems are currently known, and therefore ACT-CL J0320.4+0032 may be a rare example of a very massive halo in which quasar-mode feedback is actively taking place.
\end{abstract}

SALT Science Conference 2015 -SSC2015-

1-5 June, 2015

Stellenbosch Institute of Advanced Study, South Africa

\footnotetext{
* Speaker.
} 
You can find full details of this work published in [1].

\section{References}

[1] B. Kirk, M. Hilton, C. Cress, S. Crawford, J. P. Hughes, et al., SALT spectroscopic observations of galaxy clusters detected by ACT and a Type II quasar hosted by a brightest cluster galaxy, Monthly Notices of the Royal Astronomical Society, 449, 4010 (2015) 